\title{
An Introduction to Evidence-Based Undergraduate STEM teaching
}

\section{Professor Simon Lancaster}

An Introduction to Evidence-Based Undergraduate STEM teaching is a massive open online course running on the Coursera platform and this review is of the iteration that ran in the Autumn of 2015. The course is the product of the Center for Intergrated Research Teaching and Learning (CIRTL). The lead educators are Professors Derek Bruff and Trina McMahon. The stated objective is "to provide future STEM faculty, graduate students and post-doctoral fellows with an introduction to effective teaching strategies and the research that supports them."

The course runs over 8 weeks and is sub-divided into: principles of learning parts 1 \& 2; learning objectives; assessment; active learning, inclusive teaching; lesson planning and peer evaluation. It is structured around a top-down approach in which a course is constructed to meet clear objectives, the assessment is fit for purpose and the teaching is informed by what works and tailored to the goals.

Participants are strongly encouraged to engage and interact on the discussion forums. The Coursera platform includes an upvoting/downvoting tool which introduces a degree of gamification and will appeal to the extrinsically motivated.

The team grapple with the big issues in education and while the focus is on higher education, the American context means the topics are often at ' $A$ ' Level. What is evidence based STEM teaching? What constitutes evidence and how do we determine learning gain?

On a MOOC it is the student who owns the definition of success. Specifically, a professional educator knows when they have found what they were looking for and should then be comfortable to click away. In contrast to the FutureLearn tendency for MOOCs to serve as teaser trailers to additional content, this MOOC is a self-contained, substantial and challenging body of work and all the more rewarding for it.

This CIRTL MOOC comes highly recommended, it is not perfect and does not always practise what it preaches but it does provide an excellent introduction to an essential topic. There is an interesting discussion to be had about its value as a recognised professional development tool for STEM educators. Enrolment for the next run opens soon! 